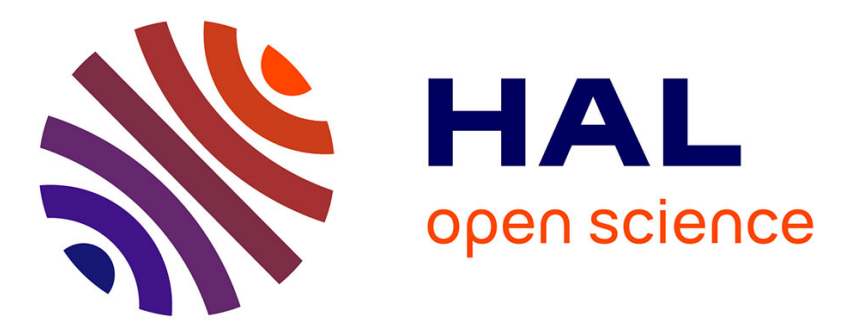

\title{
Nouvelles observations sur la stratigraphie des formations holocènes (récent) de la plaine de la Lys à Houplines (Nord)
}

\author{
Pierre-Gil Salvador, Laurent Deschodt, Sylvie Coutard
}

\section{- To cite this version:}

Pierre-Gil Salvador, Laurent Deschodt, Sylvie Coutard. Nouvelles observations sur la stratigraphie des formations holocènes (récent) de la plaine de la Lys à Houplines (Nord). Quaternaire, 2005, 16 (1), pp.65-68. 10.4000/quaternaire.243 . hal-01489474

\section{HAL Id: hal-01489474 https://hal-inrap.archives-ouvertes.fr/hal-01489474}

Submitted on 14 Mar 2017

HAL is a multi-disciplinary open access archive for the deposit and dissemination of scientific research documents, whether they are published or not. The documents may come from teaching and research institutions in France or abroad, or from public or private research centers.
L'archive ouverte pluridisciplinaire HAL, est destinée au dépôt et à la diffusion de documents scientifiques de niveau recherche, publiés ou non, émanant des établissements d'enseignement et de recherche français ou étrangers, des laboratoires publics ou privés. 


\title{
NOUVELLES OBSERVATIONS SUR LA STRATIGRAPHIE DES FORMATIONS HOLOCÈNES (RÉCENT) DE LA PLAINE DE LA LYS À HOUPLINES (Nord)
}

\author{
Pierre-Gil SALVADOR*, Laurent DESCHODT** et Sylvie COUTARD**
}

\begin{abstract}
RÉSUMÉ
Deux sondages mécaniques réalisés dans un vallon affluent de la Lys, près d'Houplines, permettent d'identifier une séquence organo-minérale d'époque gallo-romaine, traduisant une période de faible activité hydro-dynamique. Il apparaît également que l'incision des vallons dans les formations du Pléistocène supérieur est importante, supérieure à $7,70 \mathrm{~m}$, de même que le remblaiement historique qui atteint 3,30 $\mathrm{m}$ sur le site d'étude.
\end{abstract}

Mots-clés : Plaine de la Lys, Nord de la France, chronostratigraphie, Subatlantique, vallon.

\section{ABSTRACT}

NEW OBSERVATIONS ON THE STRATIGRAPHY OF HOLOCENE (MODERN) FORMATIONS OF THE LYS VALLEY AT HOUPLINES (Northern France)

Two sections digged with a mechanical excavator were opened in a tributary valley of the Lys river, close to Houplines. They allowed to identify an organomineral sequence of Gallo-Roman period, corresponding to a period of low hydrodynamic activity. It appears also that the incision of the small valleys within late Pleistocene formations is higher than 7,70 m, whereas the historical fill reaches $3,30 \mathrm{~m}$ at the same site.

Key-words : Plain of the Lys river, North of France, chronostratigraphy, Subatlantic period, small valley

A l'occasion d'une opération de sauvetage archéologique menée par l'INRAP (Marcy, 2004), deux sondages à la pelle mécanique ont été réalisés et complétés par deux datations radiocarbone. L'objet de ce court article est de présenter les nouvelles observations stratigraphiques réalisées sur ce secteur de la plaine.

\section{LE CADRE GÉOGRAPHIQUE}

La plaine de la Lys constitue au sein du Bas-Pays du Nord de la France une vaste dépression d'environ 40 km de long sur $25 \mathrm{~km}$ de large (fig. 1) dont l'origine tectonique lui confère une forme géométrique très caractéristique (Colbeaux et al., 1978 ; Sommé, 1977). D’une altitude moyenne de 18-19 m, elle est bordée dans sa partie sud-orientale par le talus rectiligne du Weppes, témoin de cette tectonique récente (Sommé, 1967). La vallée actuelle de la Lys s'inscrit à l'intérieur de cette vaste étendue, drainée par le cours historique méandriforme de la rivière aujourd'hui canalisée. Le site d'Houplines s'étend à la confluence de deux vallons de rive droite drainés par le ruisseau du Mal Attiré et le Courant du Pont Bertin, dont le cours rejoint la Lys un kilomètre plus en aval, dans la ville d'Houplines. Ces vallons s'ouvrent dans les formations limono-sableuses litées colmatant la plaine et prolongeant la zone de transition sablo-limoneuse belge (éolien). Les formations de couverture de la plaine actuelle résultent de processus nivéo-éoliens ou fluviatiles (redistribution du loess) hérités de la fin du Weichsélien. Leur épaisseur est supérieure à $5 \mathrm{~m}$. Ces formations reposent sur les argiles yprésiennes (substrat tertiaire) cartographiées approximativement vers 10 m NGF sur

\footnotetext{
* Université Lille1, UMR 8141 « Géographie des Milieux Anthropisés », Av. Paul Langevin, 59655 VILLENEUVE D’ASCQ cedex. 


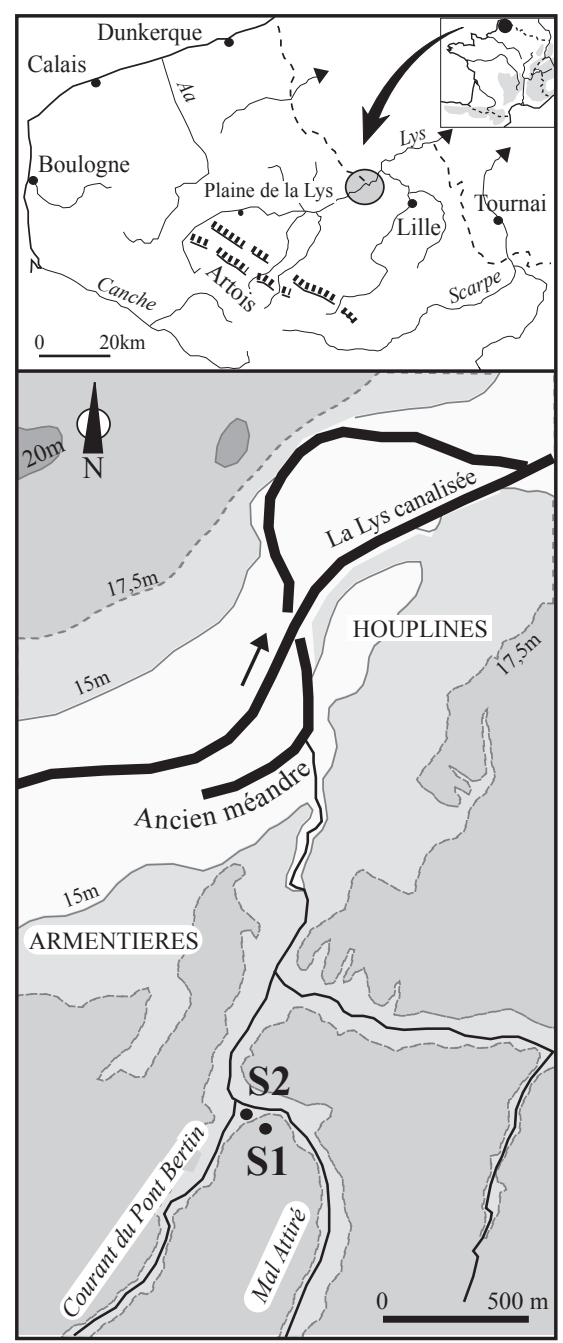

Fig. 1 : Localisation des sondages (S1, S2). Fig. 1 : Location of the corings $(S 1, S 2)$. le secteur (Sommé, 1977). L'opération archéologique a mis au jour un ensemble d'occupations protohistoriques et du début de l'époque gallo-romaine, implantées sur les versants des vallons affluents.

\section{DESCRIPTION ET INTERPRÉTATION DES SONDAGES}

Deux sondages ont été réalisés, en position haute et dans le fond du vallon du " Mal Attiré », sur une profondeur de 4 à $5 \mathrm{~m}$ (fig. 2). Ils sont séparés par un versant nettement convexe caractéristique des cours d'eau drainant la plaine.

Le sondage 1 en position haute (18,60 m IGN 69) est ouvert dans les formations limono-sableuses litées. La partie superficielle est altérée par la pédogenèse - points de manganèse, traces d'oxydation, litage non visible mais la structure litée apparaît rapidement, dès $80 \mathrm{~cm}$ de profondeur, sous la forme d'une alternance de lamines millimétriques de sables fins et de limons plastiques. Des traces de malacofaunes dans les niveaux limoneux sont observées. Des agrégats limoneux dont l'angulosité traduit une structuration cryogénique sont également repérés au sein des laminations (reprise fluviatile de lamines antérieures ?). A la base, les lamines s'épaississent et des reprises érosives sont repérées. En lame mince, les laminations sont nettes, souvent ondulées et d'épaisseur variable (photo 1). Elles correspondent à des séquences au granoclassement normal depuis des sables fins jusqu'à des argiles silteuses poussiéreuses (décantation). La base des séquences, grossière, est composée de sables fins propres (quartz, micas...), en contact généralement érosif avec la séquence limono-argileuse précédente, dont le sommet est démantelé et repris sous

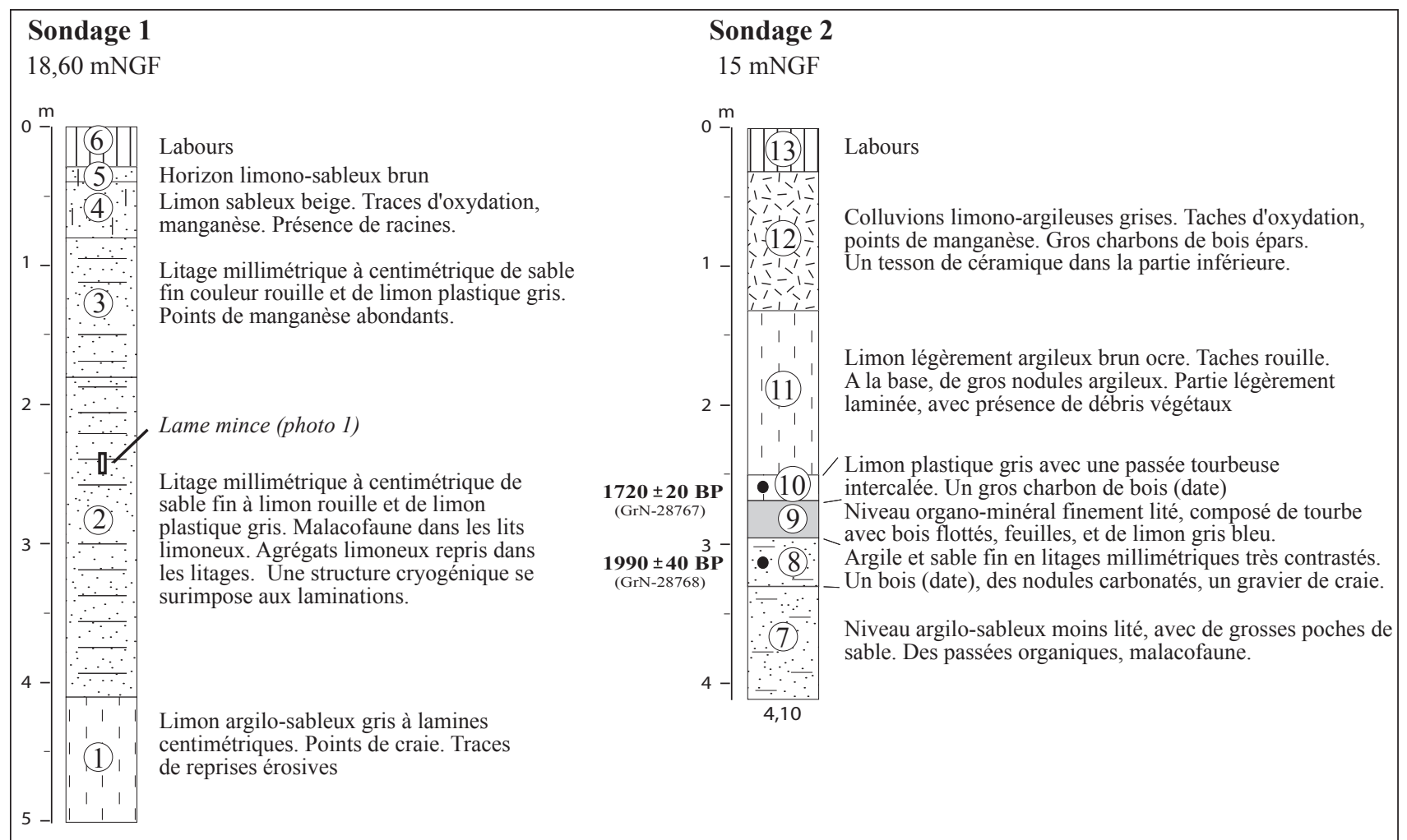

Fig. 2 : Logs stratigraphiques en haut de versant (sondage 1) et fond de vallon (sondage 2).

Fig. 2 : Stratigraphical profiles at the top of the slope (coring 1) and in the talweg (coring 2). 


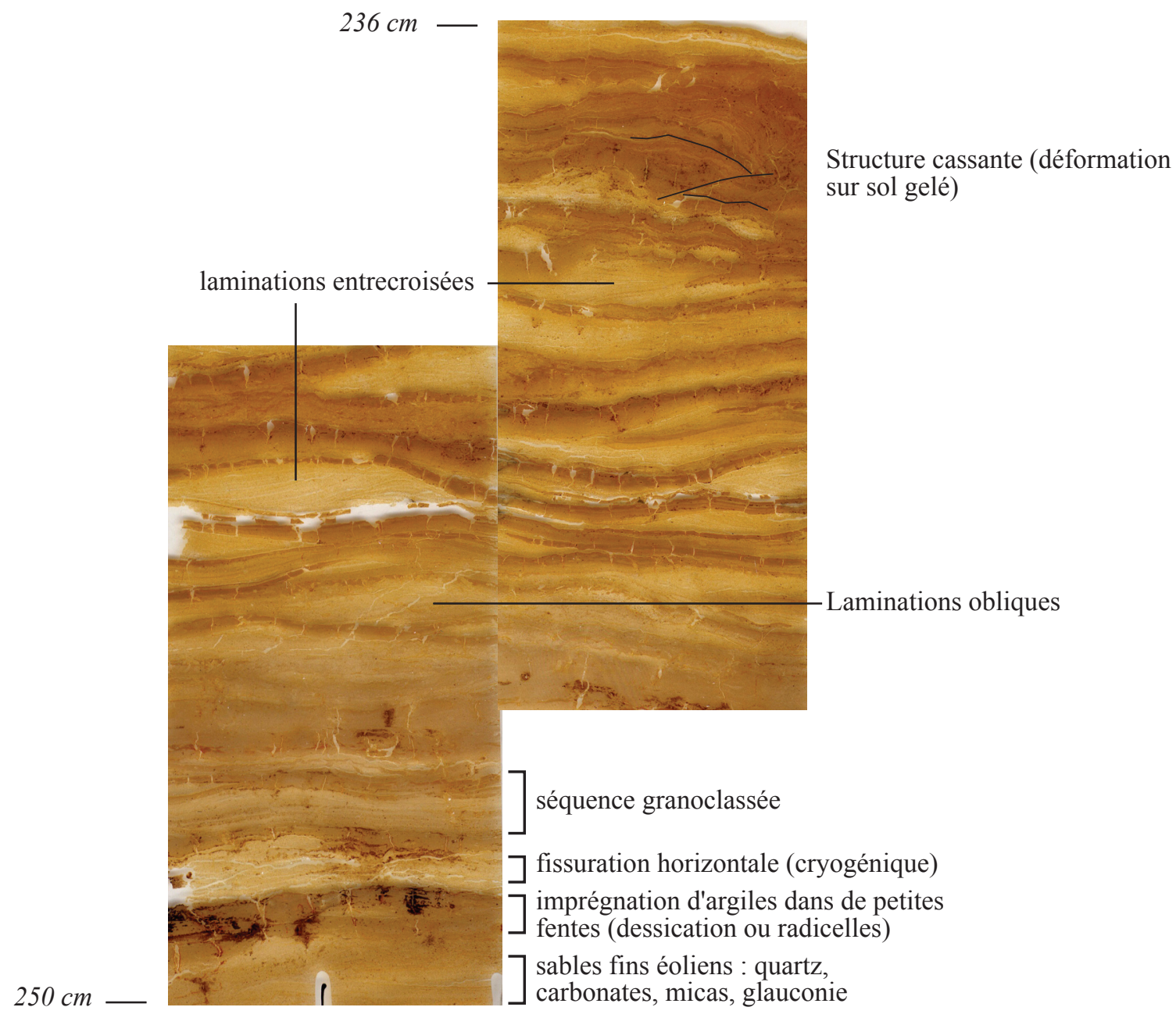

Photo 1 : Analyse micromorphologique d'une partie des formations limono-sableuses litées (sondage 1). Photo 1 : Micromorphology analysis of a part of the laminated silty sand deposits (coring 1).

forme d'agrégats plus ou moins roulés. La présence de laminations obliques et entrecroisées, la réorientation des paillettes de micas parallèlement aux lamines, la présence de vésicules arrondies secondairement comblées par des grains grossiers dans les niveaux fins (mise en place à l'état boueux) témoignent d'un dépôt par ruissellement et écoulement laminaire (Mücher et De Ploey, 1977). Cette reprise et redistribution des dépôts éoliens peuvent être parfois associées aux fontes printanières. L'impact du gel se marque par des fissures horizontales parfois imprégnées d'argiles et des déformations cassantes. Ces observations traduisent une ambiance environnementale froide où prédominent les processus périglaciaires.

Le sondage 2 en position de fond de talweg $(15,00 \mathrm{~m}$ IGN 69) présente une stratigraphie plus diversifiée et reflète les conditions topographiques locales. Le sommet de la séquence est associé à des colluvions, dans lesquels de gros charbons de bois et un tesson de céramique ont été repérés. Ces dépôts de versant peuvent être raccordés à titre d'hypothèse à une érosion d'origine anthropique résultant de l'occupation protohistorique et galloromaine développée sur le secteur. La section inférieure est plus originale. Des niveaux limoneux gris plastiques et un petit niveau tourbeux d'environ $30 \mathrm{~cm}$ d'épaisseur ont été mis au jour entre $2,50 \mathrm{~m}$ et $3 \mathrm{~m}$ de profondeur. Ils indiquent un environnement de type palustre, inscrit dans le fond du talweg. L'ensemble est supporté par un niveau très particulier d'environ $40 \mathrm{~cm}$ d'épaisseur, caractérisé par une alternance régulière de niveaux limono-argileux et sableux bien contrastés, d'épaisseur millimétrique (photo 2). Son interprétation reste problématique. Le niveau de base, plus grossier et moins lité, indique une mise en place dans des conditions plus dynamiques.

\section{DISCUSSION ET CONCLUSION}

Le sondage effectué au sommet du versant n'apporte pas d'élément nouveau à la connaissance de la plaine en décrivant les formations superficielles limono-sableuses litées qui la recouvrent. L'intérêt principal des observations est contenu dans la séquence localisée dans le fond du vallon, en raison des deux datations obtenues à $2,60 \mathrm{~m}$ de profondeur sur un charbon de bois $(1720 \pm 20$ $\mathrm{BP}, 250(95,4 \%) 400 \mathrm{AD}, \mathrm{GrN} 28767)$ et à $3,20 \mathrm{~m}$ sur un bois flotté (1990 $\pm 40 \mathrm{BP}, 100 \mathrm{BC}(95,4 \%)$ 130AD, GrN28768). Ces résultats placent l'ensemble des niveaux finement lités (8) et la séquence organo-minérale majeure (9-10) à l'époque gallo-romaine. Le colmatage identifié du vallon est ainsi caractérisé par un hydrodynamisme décroissant durant cette période, avant que ne 


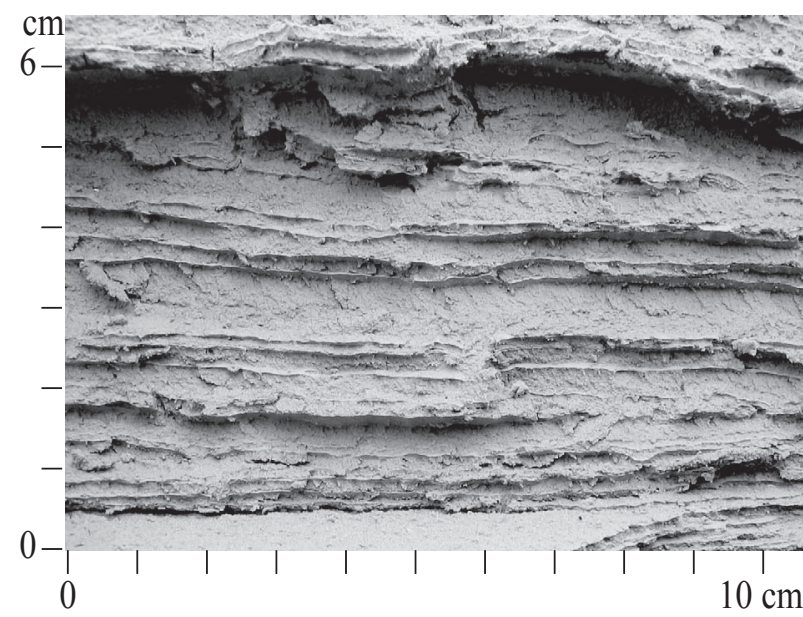

Photo 2 : Détail des laminations du niveau 8. Photo $2:$ Detail of the laminated deposits from unit 8 .

lui succède une phase associée à des apports plus grossiers et à dominante minérale (limons d'inondation du niveau 11). L'interprétation du dépôt (8) à microlaminations très contrastées pose problème. On peut l'envisager comme la conséquence naturelle d'un effet de confluence lié à la proximité de la Lys, avec un processus régulier d'inondation par refoulement. Néanmoins, le dépôt présente un faciès très différent des limons d'inondation repérés habituellement dans ces fonds de vallée et auquel correspond plus sûrement le niveau 11. L'hypothèse d'un dépôt dont la mise en place est sous contrôle anthropique mérite d'être envisagée, en liaison avec le fonctionnement d'un aménagement hydraulique. Un faciès proche (alternance de sable et de limon très régulièrement lités) a déjà été observé dans la vallée de la Somme à Etouvie, immédiatement en aval d'un bassin de rétention des eaux, et expliqué par la vidange régulière de l'ouvrage (Deschodt et Harnay, 1997).
On notera également l'importance du colmatage historique de ce vallon, de l'ordre de 3,30 m pour les deux derniers millénaires. La puissance de ce remblaiement met en valeur l'importance de l'incision des vallons élémentaires dans les sédiments hérités du Pléistocène supérieur, plus de 7,70 $\mathrm{m}$ dans ce secteur de la plaine (de la surface de la plaine à la base du sondage réalisé dans le vallon), que l'on pourrait mettre en rapport avec le léger creusement tardi et post-glaciaire de la Lys observé en aval d'Armentières.

Bien évidemment, le caractère ponctuel des données ne prête pas à la généralisation, d'autant qu'elles concernent un segment élémentaire du réseau hydrographique. Mais c'est également là tout l'intérêt de ce type d'investigations dont la multiplication permet une approche plus globale des processus à l'échelle du bassin versant.

\section{RÉFÉRENCES}

COLBEAUX, J.-P., LEPLAT, J., PAEPE, R. \& SOMMÉ, J., 1978 Tectonique récente dans le Nord de la France et le Sud de la Belgique : exemple de la Plaine de la Lys (Feuille d'Hazebrouck à 1/50 000), Annales de la société géologique du Nord, tome XVCVIII, séance du 9 mars 1978, 179-188.

DESCHODT, L. \& HARNAY, V., 1997 - Amiens-étouvie (Somme) «Le Chemin de la Marine»1996, Centre d'entretien SANEF, Analyse stratigraphique des dépôts fluviatiles holocènes et aménagements hydrauliques gallo-romains - DFS, AFAN Centre-Nord avec le concours de la SANEF, SRA Picardie, 2 t., 204 p.

MARCY, T., 2004 - Houpline. " ZAC d'Houplines-Mal Attiré ». Rapport de diagnostic, INRAP Nord-Picardie, SRA Nord-Pas-de-Calais, $29 \mathrm{p}$.

MÜCHER, H.-J. \& DE PLOEY, J., 1977 - Experimental and micromorphological investigation of erosion and redeposition of loess by water. Earth Surface Processes and Landforms, 2, 117-124.

SOMMÉ, J., 1967 - Tectonique récente dans la région de Lille : pays de Weppes et Mélantois occidental, Revue de Géomorphologie Dynamique, $\mathrm{n}^{\circ} 2,11 \mathrm{p}$.

SOMMÉ, J., 1977 - Les plaines du Nord de la France et leur bordure. Etude géomorphologique. Thèse d'Etat, Univ. Paris I, 1975, Paris, Champion, 2 vol. : 1-810, 185 fig. 